\title{
Propuesta de un programa de tratamiento para dejar de fumar basado en la Activación Conductual
}

\author{
Johanna Sánchez \\ Michel Reyes \\ Instituto de Ciencia y Terapia Conductual, México
}

\author{
Jorge Barraca \\ Universidad Camilo José Cela, España
}

\author{
Jazmín Mora \\ Instituto Nacional de Psiquiatría "Ramón de la Fuente Muñiz", México
}

\section{RESUMEN}

El objetivo del presente artículo es proporcionar a la comunidad científica y a los psicólogos aplicados un programa para dejar de fumar basado en la Activación Conductual en México. Con el fin de que pueda replicarse en distintos países y contextos, se anexa el material necesario para cada sesión, así como sus técnicas, procedimientos y puntos claves que considerar. Los resultados de la intervención, indican que el programa de activación conductual tuvo un efecto prometedor para reducir el consumo de tabaco en este grupo y puede ser un recurso útil replicado en países de habla hispana y particularmente en América Latina. No obstante, se requiere ampliar en investigación en esta área para aportar evidencia de su eficacia. Estos hallazgos, llevan a los autores a discutir y concluir los puntos fuertes y barreras que tener presentes en su implementación.

Palabras clave

Activación Conductual; programa de tratamiento; dependencia tabáquica.
ABSTRACT

The objective of this article is to provide the scientific community and applied psychologists with a smoking cessation program based on Behavioral Activation in Mexico. In order to replicate in different countries and contexts, the necessary material for each session is annexed, as well as its techniques, procedures and key points to consider. The results of the intervention indicate that the behavioral activation program had a promising effect to reduce tobacco consumption in this group and may be a useful resource replicated in Spanish-speaking countries and particularly in Latin America. However, it is necessary to expand research in this area to provide evidence of its effectiveness. These findings lead the authors to discuss and conclude the strengths and barriers to keep in mind in their implementation.

KeYWORDS

Behavioral Activation; treatment program; tobacco dependence. 
El consumo de tabaco es un grave problema de salud pública en el mundo. De acuerdo con los datos más recientes de la OMS, es el responsable de la muerte de más de ocho millones de personas al año, de las cuales siete son consumidores directos (OMS, 2019). En México, la última Encuesta Nacional de Consumo de Drogas, Alcohol y Tabaco (ENCODAT, 20162017) ha revelado que el $17,6 \%$ de la población de 12 a 65 años fuma tabaco actualmente, lo que se significa un total de 14,9 millones de mexicanos (Villatoro-Velázquez et al., 2017).

Este problema se complica, si además coexiste con síntomas depresivos y ansiosos. De hecho, las personas con síntomas de depresión y/o ansiedad, tienen más probabilidades de fumar o de continuar haciéndolo cuando intentan dejarlo (Leventhal \& Zvolensky, 2015).

Dada entonces la comorbilidad de los diagnósticos y la frecuencia de estos, es necesario el desarrollo de intervenciones que permitan abordar estos diagnósticos simultáneamente (Martínez-Vispo, Martínez, López-Durán, Fernández del Río, \& Becoña, 2018; Mathew, Hogarth, Leventhal, Cook, \& Hitsman, 2017).

En este sentido, la Activación Conductual (AC) figura como una de las opciones más prometedoras en el tratamiento de tabaquismo comórbido con la sintomatología mencionada (Leventhal \& Zvolensky, 2015). Es una intervención desarrollada inicialmente para la depresión que se enfoca en aumentar la participación en actividades agradables (Mckay, 2016). Tiene sus raíces teóricas en Ferster (1973) y Lewinsohn (1974), y creció su interés a partir del trabajo de Jacobson et al. (1996), quienes de manera aleatoria asignaron a 150 personas con depresión a tres condiciones: grupo AC; AC y reestructuración cognitiva, incluyendo pensamientos automáticos relacionados con la hipótesis de afrontamiento; y Terapia Cognitiva (TC) completa (AC, pensamientos automáticos, reestructuración cognitiva y modificación de las creencias básicas), relacionada con la hipótesis cognitiva, se pudo observar que no había diferencias significativas tanto clínicas, como estadísticas entre los tres grupos y llegaron a la conclusión de que los componentes cognitivos de la terapia eran redundantes. Esto demostró que la TC completa no proporcionó mejores resultados que la reestructuración cognitiva o la activación conductual. Además, esta eficacia se mantuvo durante los dos años de seguimiento (Gortner, Gollan, Dobson, \& Jacobson, 1998).

A partir de estos hallazgos, se han realizado varios ensayos clínicos aleatorizados (ECA), en donde se ha concluido que la $A C$ es una alternativa viable, eficaz y costo-efectiva, equiparable a la Terapia Cognitivo Conductual (TCC) y a la medicación en personas con depresión (Dimidjian et al., 2006; Dobson et al., 2008).

Otros estudios en menor escala también han mostrado que AC es efectiva (Collado, Castillo, Maero, Lejuez, \& MacPherson, 2013; Kanter et al. 2014; Shared, 2016). Recientemente se ha despertado el interés por aplicar AC en pacientes con depresión y tabaquismo (Aldi, Bertoli, Ferraro, Pezzuto, \& Fiammetta, 2018; Busch et al. 2017; Collado, Calderón, MacPherson, Lejuez, 2016; Cooper, Borland, Mckee, Yong, Dugue, 2016; MacPherson, Collado, Ninnemann, \& Hoffman, 2017; MacPherson et al. 2010; Mathew, Hogarth, Leventhal, Cook, \& Hitsman, 
2017; Secadez-Villa, Vallejo-Seco, García-Rodríguez, López-Nuñez, Wiedberg, González-Roz, 2015); y la intención es extender los hallazgos a pacientes que además de fumar tabaco y presentar sintomatología de depresión, también muestren sintomatología ansiosa.

El objetivo del presente artículo es proporcionar un programa diseñado desde la Activación Conductual para dejar de fumar. Este programa fue fundamentado en tres principales textos: El Manual de tratamiento revisado, de Lejuez, Hopko, Acierno, Daugthers, \& Pagoto (2011), el manual de Martell, Dimidjian y Hermann-Dunn (2010), y el manual para el tratamiento de la depresión de Barraca y PérezÁlvarez (2015).

\section{Programa de AC para el cese de la conducta tabáquica}

El objetivo del programa fue identificar actividades reforzantes naturales vinculadas a las áreas de vida valiosas de las participantes e incorporarlas progresivamente a su rutina diaria con el fin de mejorar los síntomas de depresión y ansiedad, y lograr la abstinencia sostenida del consumo de tabaco. El programa presentado está diseñado para ser aplicado por el terapeuta.

Antes de describirlo se repasan a continuación aspectos generales para aplicar la intervención en el ámbito de tabaquismo.

\section{Aspectos generales del programa}

El programa incluyó 1 sesión de evaluación, 10 sesiones grupales de 90 minutos ( 2 sesiones semanales) y 4 sesiones de seguimiento (1, 3 , 6 meses).
De la sesión 1 a la 2 (primera semana), se realiza el monitoreo de consumo, emoción y actividad asociada. La abstinencia comienza a partir de la sesión 3 (el día 7).

Sesión de evaluación o pretratamiento: Se realizó una entrevista semiestructurada que contiene información sobre datos del paciente, historia de tabaquismo, estado actual de consumo, datos médicos, consumo de tabaco, historia de depresión e historia de ansiedad. Se aplica el Inventario de Depresión de Beck II, versión adaptada y validada en México (González, Reséndiz, \& Reyes-Lagunes, 2015), el Inventario de Ansiedad de Beck, versión adaptada a México por Robles, Varela, Jurado, \& Páez (2001), la Escala de Activación Conductual, versión adaptada por Sánchez-Angulo, Barraca, Mora, \& Reyes-Ortega (2018) y la versión abreviada del Test de Fasgeström (Becoña \& Vázquez, 1998).

El material usado es adaptado por los autores, pero desarrollado por Martell et al. (2010).

Al finalizar la intervención se mide el patrón de consumo a través del CO exhalado y se aplica la Escala de Activación Conductual, el Inventario de Depresión de Beck II y el Inventario de Ansiedad de Beck para comparar los puntajes entre la medición inicial y la medición final.

La medición anterior se repite durante los seguimientos. Se recomienda realizar seguimiento de por lo menos 1 año.

El programa se enmarca en técnicas como programación y monitoreo de actividades flexibles, fragmentación y jerarquización de actividades, exposición a situaciones evitadas, ensayo verbal de la tarea, análisis funcional de la conducta, registro conductual, ensayo con- 
ductual y el análisis de barreras con enfoque de solución de problemas. Su foco es encaminar a la acción y trabajar con perspectiva de solución de problemas con las barreras que se presenten.

\section{Descripción del Programa}

\section{Sesión 1. Introducción de la intervención}

Objetivo: Lograr que se comprenda el fundamento de AC y su relación con el consumo de tabaco.

Puntos de la sesión:

Presentación del grupo.

Delimitación de los objetivos.

Firma contrato terapéutico.

Identificación de los motivos para dejar de fumar.

Análisis funcional de las situaciones relacionadas al tabaquismo.

Ensayo conductual del registro de consumo y actividades.

Identificar barreras para realizar la tarea.

Medición de CO exhalado.

Cierre de la sesión.

Material necesario para la sesión.

Modelo de activación conductual (ver Martell et al., 2010).

Folleto con los 10 principios de la Activación Conductual (ver Martell et al., 2010).

Contrato terapéutico.

Registro de monitoreo de consumo y actividades (ver anexo1).

Guía de preguntas de acuerdo con la lógica de AC (ver anexo 2).

Hoja para explorar barreras (ver Martell et al., 2010).
Técnicas, ejercicios y procedimiento.

Presentación del grupo. Además de mencionar su nombre y preguntar el de los participantes, el o la terapeuta deberá realizar dos preguntas claves para iniciar la sesión: tiempo fumando y número de cigarrillos. Estas preguntas le ayudarán a construir el perfil general del grupo y así conducir con mayor facilidad la sesión.

Delimitación de los objetivos y conducta meta. Una vez anotados en la pizarra el tiempo de consumo y número de cigarrillos al día, el terapeuta proporciona una explicación general sobre las 10 sesiones de intervención estableciendo el día de abstinencia y la meta.

Medición de CO exhalado. Se realizó una medición inicial del CO exhalado y se usó esta información para motivar a los pacientes a dejar de fumar, relacionándolos a los beneficios al dejar de hacerlo. Se destaca lo positivo más que las consecuencias de seguir fumando.

Identificación de los motivos para dejar de fumar y las dificultades para hacerlo. Se exploró a través de la conversación guiada los motivos que tiene el grupo para dejar de fumar y se anotan en la pizarra. Aspecto este importante de tener en cuenta durante todas las sesiones para que funcione como motivador al cambio conductual. Además, se identificaron las dificultades que se han presentado hasta ahora en cada participante al momento de intentar de dejar de fumar. Esto último, con el fin de recuperar esa información y trabajarlo como barreras para dejar de fumar, enfocándose en la solución de problemas.

Análisis funcional de la situación depresiva/ ansiosa y su relación con el consumo. El tera- 
peuta se apoya de algún recurso visual para explicar gráficamente el análisis funcional, centrándose en las situaciones de depresión y ansiedad y su relación son el consumo de tabaco. Es importante durante el análisis que se use el modelo de activación conductual propuesto por Martell et al. (2010). La forma de presentar el modelo es el siguiente: a el terapeuta le corresponde explicar cómo la persona termina en una situación depresiva y ansiosa, y cómo lo que hace mantiene la situación. Es necesario que la persona ubique el consumo de tabaco como una de las conductas de evitación para escapar de la sintomatología, lo que a la larga no cambia la situación depresiva, y la necesidad de hacer cosas diferentes con el fin de salir de ella. Para ello, se llevó a discusión al menos una experiencia de algún participante del grupo en la que el resultado de la conducta haya sido el mantenimiento de la depresión/ansiedad y el consumo de tabaco haya estado involucrado. Una vez que el modelo quede ejemplificado y claro, se presenta el objetivo de la intervención y los 10 principios fundamentales de la activación conductual (ver Martell et al., 2010).

Ensayo conductual del registro de consumo y actividades. El terapeuta, a través del registro del monitoreo de actividades, realizó un ensayo para registrar el último consumo de tabaco y las actividades realizadas durante la semana. Además de la actividad registrada, se tomará en cuenta el placer o disfrute de esa actividad $(\mathrm{P})$, el valor o importancia (V) y la competencia o eficacia para realizarla (C). Este ensayo ayudará a que las participantes lo realicen como tarea durante la semana.
Identificar barreras para realizar la tarea. El terapeuta se guía con la lista de preguntas para identificar las cosas que pueden salir mal. Utilizará la hoja Trigger, Response, Avoidance-Pattern (TRAP) para analizar las barreras y desarrollar estrategias para manejarlas (ver Martell et al., 2010).

Cierre de la sesión. Se preguntan las dudas y se recuerda la importancia de realizar el registro de tarea, incluso como una forma de activación.

Aspectos que debe cubrir el terapeuta

Aclarar dudas.

Emplear un formato visual del modelo conductual para desarrollar una conceptualización del patrón de consumo, depresivo y ansioso de las participantes como grupo.

Explicar que los acontecimientos que suceden influyen en la experiencia y en el impacto de la depresión y la ansiedad.

Explicar que la depresión se caracteriza por un patrón de pasividad y/o de evitación.

Explicar que la depresión y la ansiedad no es culpa del individuo y que sentirse así y actuar de forma pasiva/evitativa tiene sentido (validar la experiencia).

Explicar que la programación de actividades es el corazón de la terapia y que en esta se busca un cambio de fuera hacia dentro. Se refiere a que el primero es actuar para luego generar un cambio en lo que se siente.

Enfatizar la importancia de realizar las actividades para desarrollar un patrón de vida saludable.

Explicar que el objetivo de la terapia es construir un patrón conductual que permita aumentar la sensación de bienestar, importancia y competencia. 
Hay que explicar que la activación es progresiva y no de seguir una lógica de todo o nada.

Entregar folleto con los 10 principios básicos de la AC.

Explicar que la terapia se adapta al ritmo de cada participante.

Realizar un ensayo conductual para el monitoreo de actividades, empleando como ejemplo el día anterior.

Explicar que la terapia se inicia con el análisis de las actividades que realizan o que realizaban y dejaron de realizar.

Explicar la necesidad de completar el grado de placer, competencia y valor (o importancia) en los registros.

Explorar barreras internas (olvidos, rumiación, comprensión, otras reacciones emocionales), externas (otras personas, situación, apoyo social) y de implementación (conflictos para realizar la tarea, riesgos, grado de dificultad, habilidades para realizarla, recursos necesarios).

Recomendaciones para el terapeuta

No se trata de una exposición teórica del modelo y de utilizar lenguaje técnico, sino de representar gráficamente el modelo con la experiencia que presente el grupo.

Realizar la medición de CO al final de la sesión.

La comprensión del modelo es clave para la intervención, ya que su aceptación otorga sentido a la programación y monitoreo de actividades.

Dificultades que se deben anticipar

Poca comprensión del modelo. Para ello es necesario utilizar tanta cantidad de experiencias grupales como sean posibles.
Argumentos en contra de no poder completar la tarea por falta de tiempo. Aportar soluciones en colaboración con el paciente que permita la realización de estay enfatizar que la forma de llegar al objetivo de dejar de fumar es realizar la tarea.

\section{Sesión 2. El tabaquismo y las emociones}

Objetivo. Comenzar programación de actividades.

Puntos de la sesión

Revisión del registro de consumo y actividades.

Revisión de las puntuaciones más altas de placer, valor y competencia.

Discusión sobre las actividades relacionadas al consumo y aquellas libres de consumo.

Programación de actividades, a partir del día de hoy hasta la próxima sesión.

Entregar el registro de la tarea.

Inicio de la abstinencia.

Material necesario para la sesión

Registro de monitoreo de consumo y actividades realizado en casa (ver anexo 1).

Guía de preguntas de acuerdo con la lógica de AC (ver anexo 2).

Programación de actividades (ver Martell et al., 2010).

Técnicas, ejercicios y procedimiento

Revisión del registro de consumo y actividades. El terapeuta, con ayuda del formato de monitoreo de actividades que se llevaron de tarea, identifica a través de preguntas ¿quién realizó la tarea?, ¿cuáles fueron las dificultades? Y recuerda las estrategias que plantearon en la sesión anterior como recursos para afrontarlas. Utiliza refuerzo social y valora el intento, 
pero recuerda que la efectividad de la estrategia depende de hacer. A aquellas personas que no la realizaron se les preguntan cuatro aspectos claves: ¿fue un problema de olvido?, ¿de comprensión?, ¿fue un problema de conflicto de actividades?, ¿fue un problema de complejidad? Y para mayor profundización el anexo 2 tiene preguntas para identificar aspectos más específicos de cada una de esas preguntas. Se selecciona al menos una persona para analizar el consumo, la actividad que la acompañaba, así como el placer, el valor y la sensación de competencia en cada una. Este ejercicio permite identificar las actividades que más se repite en relación con el consumo, la emoción y los dominios de realizarlas, y las que no; anticipando aquellas actividades que no deberían programarse durante la activación. Sin embargo, si es una actividad que no puede prescindir, como por ejemplo el trabajo, es necesario sustituir la conducta de fumar por otra.

Revisión de las puntuaciones más altas de placer, valor y competencia. Se les pide a quienes realizaron la tarea que identifiquen y marquen con un color (entrega marcadores) los 3 puntajes más altos de valor o importancia (V). Se apoya de un recurso visual como la pizarra para organizar las categorías que encierran las actividades desempeñadas por las participantes durante la semana. Se identifican los puntajes de valor más altos, antes de los de placer y competencia; partiendo del supuesto que a pesar de no disfrutar la actividad o sentir agrado, es relevante hacer cosas importantes para los participantes. Este dato se retomará al momento de programar las actividades.

Discusión sobre las actividades relacionadas al consumo. En este punto se enfatizan el consumo y el contexto en el que se desarrolla. Es importante realizar dos preguntas claves al respecto: 1) ¿qué actividades observan que más se repite mientras se fuma?, 2) ¿Qué puede estar disparando el consumo durante esa actividad?

Programación de actividades, a partir del día de hoy hasta la próxima sesión. Partiendo de las categorías de actividades identificadas en la revisión del registro, se les pedirá a los participantes que programen diez actividades objetivo y que seleccionen al menos una en su caso particular (pueden usar las categorías identificadas para orientarse). Para ello, se pregunta ¿qué actividades ya has realizado antes y has abandonado? ¿Cuáles de esas tiene un valor alto para ti? ¿Qué actividad sería valiosa para ti realizar y que no hayas podido hacerlo? Se recomienda analizar actividades que hayan realizado antes y luego otras que nunca hayan hecho y deseen hacerlo. Es importante desglosar aquellas actividades que sean más complejas, teniendo presente que es necesario convertirlas en pequeñas actividades semanales.

Para la programación de actividades, el terapeuta se apoya en el material (ver Martell et al., 2010), en donde se seleccionarán las actividades que realizar a partir de hoy y hasta la próxima sesión. Para ello es necesario saber el grado de dificultad y la viabilidad de la actividad, así como los recursos para su realización. Es importante identificar cuándo la persona utiliza alguna actividad como evitación de consumo y no como una fuente de reforzamiento positivo.

En este punto, se deben generar por lo menos cinco actividades de acuerdo con las categorías y aspectos comunes. Las actividades 
deben cubrir un amplio rango y deben ser actividades que puedan dar placer inmediato $y$, de no ser posible, utilizar el hetero-reforzamiento (a través de redes de apoyo sociales). Se deben motivar actividades que sean incompatibles con el consumo de tabaco, debido a que se encuentra en la fase de preparación para dejar de fumar (comienza la abstinencia), incluyendo actividades que impliquen movilización corporal y considerar actividades previas y que figuren entre las primeras actividades que ejecutar. El nivel de movilización corporal dependerá del caso.

Entrega del registro de consumo. Nuevamente se entrega el registro de consumo.

Inicio de la abstinencia a partir de la próxima sesión. Se explica que a partir de la sesión siguiente comienza la abstinencia al consumo y que, por lo tanto, a partir de ese día la consigna es no fumar. Se discuten los diferentes fármacos para dejar de fumar como bupropión y los parches, y se entrega las indicaciones de acuerdo con cada persona.

Aspectos que debe cubrir el terapeuta

Recordar que las actividades se realizarán en función de un plan y no de una emoción.

Enfatizar la participación grupal sobre el análisis del problema individual.

Evaluar las barreras externas, internas y de implementación (TRAP).

Identificar categorías y aspectos comunes de las actividades.

Generar un mínimo de dos actividades de acuerdo con las categorías de actividades antidepresivas del grupo.

Evaluar con el grupo el nivel de dificultad para realizar las actividades seleccionadas.
Valorar la viabilidad de realizar la actividad seleccionada.

Graduar las actividades extensas de acuerdo con los pasos en que se pueden dividir.

Valorar los riesgos para las participantes (actividades físicas que pudieran atentar en contra de la salud, como ejercicio físico que implique un nivel de esfuerzo no recomendado por el médico).

Evaluar los recursos internos y externos para realizar las actividades seleccionadas.

Generar estrategias para resolver los problemas identificados por el grupo.

Plantear y responder a preguntas de comprensión de la actividad.

Dificultades que se deben anticipar

Programar actividades que deseen pero que no sean probables o viables.

Plantear la posibilidad de que no exista ninguna actividad.

Actividades que estén relacionadas al consumo y que no puedan prescindirse.

\section{Sesión 3. Situaciones de riesgo y activación conductual}

Objetivo. Identificar las situaciones que promueven el consumo y programar actividades incompatibles al consumo.

Puntos de la sesión.

Revisión del registro de consumo y actividades.

Revisión de las puntuaciones más altas de placer, valor y competencia.

Revisión de las actividades.

Análisis de las situaciones de riesgos.

Programación de actividades, a partir del día de hoy hasta la próxima sesión.

Entregar el registro de la tarea. 
Material necesario para la sesión

Registro de monitoreo de consumo y actividades realizado en casa (ver anexo 1).

Guía de preguntas de acuerdo con la lógica de AC (ver anexo 2).

Formato de programación de actividades (ver Martell et al., 2010).

Técnicas, ejercicios y procedimiento.

Revisión del registro de consumo y actividades. Se repite el procedimiento de la sesión anterior. Sin embargo, se enfatiza que ya no habrá registro porque comienza la abstinencia a partir de ese momento. Se identifican aquellas personas que no lograron comenzar la abstinencia, así como las barreras para lograrlo, con el fin de realizar un plan de acción que contribuya al logro de esta partir de ahora.

Revisión de las puntuaciones más altas de Placer, Valor y Competencia. Se repite el procedimiento de la sesión anterior.

Revisión de las actividades planeadas. Se revisa la realización de las actividades planteadas en la sesión anterior, y las barreras en caso de no completarlas. Se apoya del anexo 4 , en donde se preguntan aspectos de olvido, dificultad, comprensión y conflicto de actividades. Se debe revisar si realmente las actividades que escogieron son viables.

Análisis de las situaciones de riesgos. Se discuten las estrategias a utilizar cuando se enfrenta a situaciones comunes de alto riesgo, incluyendo estado de ánimo negativo y bajo efecto positivo, utilizando un marco de AC, el cual propone la activación como una forma de manejar las situaciones de riesgos.

Programación de actividades, a partir del día de hoy hasta la próxima sesión. Se repite el procedimiento de la sesión anterior. Se puede continuar con las actividades planeadas y/o incluir alguna otra, esto dependerá de la persona. Si la persona logró realizar la actividad de la semana anterior con éxito, incorporará otras actividades, si no se analizará si esta actividad necesita descomponerse en pasos para ser incluida para la semana o cambiarse.

Entregar el registro de la tarea.

Aspectos que debe cubrir el terapeuta.

Motivar actividades que sean incompatibles con el consumo de tabaco, incluyendo actividades que impliquen movilización corporal y considerar actividades previas y que figuren entre las primeras actividades a ejecutar. El nivel de movilización corporal dependerá del caso. Resolver dudas y cerrar con el aprendizaje del día de hoy.

Cubrir la mayor cantidad de situaciones de riesgos posibles.

Dificultades que se deben anticipar

Que no encuentren placer, valor y/o competencia en las actividades que creyeron beneficiosas y que programaron anteriormente.

Que se planteen actividades ambiguas y poco viables.

Que no muestren interés en la realización de la actividad.

\section{Sesiones 4 a 9. Activación conductual como plan de acción}

Objetivo. Incorporar actividades reforzantes a sus hábitos de vida incompatibles con el consumo

Puntos de la sesión

Monitoreo de la abstinencia.

Revisión de las actividades programadas. 
Programación de actividades objetivo para la semana.

Materiales necesarios para la sesión

Formato de programación de actividades (ver Martell et al., 2010).

Técnicas, ejercicios y procedimientos

Monitoreo de la abstinencia. Al iniciar la sesión se valoran los esfuerzos para dejar de fumar y lo que han logrado hasta ahora, los beneficios percibidos y las desventajas de dejar de fumar, las razones por las que decidieron dejar de fumar, y los efectos secundarios relacionados con el uso del fármaco para dejar de fumar. Asímismo, se discuten las situaciones de alto riesgo durante la semana y las formas de enfrentarlas.

Revisión de las actividades programadas. Se revisa la realización de las actividades planteadas en la sesión anterior, y las barreras en caso de no completarlas. Se apoya del anexo 4, en donde se preguntan aspectos de olvido, dificultad, comprensión y conflicto de actividades. Se debe revisar si realmente las actividades que escogieron son viables.

Programación de actividades objetivo para la semana. Se repite el procedimiento de la sesión dos. Se puede continuar con las actividades planeadas y/o incluir alguna otra, esto dependerá de la persona. Si la persona logró realizar la actividad de la semana anterior con éxito, incorporará otras actividades, sino se analizará si esta actividad necesita descomponerse en pasos para ser incluida para la semana o cambiarse.

Aspectos que debe cubrir el terapeuta

Recordar que las actividades se realizarán en función de un plan y no de una emoción.
Enfatizar la participación grupal sobre el análisis del problema individual.

Evaluar las barreras externas, internas y de implementación (TRAP) (ver Martell et al. 2010).

Dificultades que se deben anticipar

Que no encuentren placer, valor y/o competencia en las actividades que creyeron beneficiosas y que programaron anteriormente.

Que se planteen actividades ambiguas y poco viables.

Que no muestren interés en la realización de la actividad.

\section{Sesión 10. Prevención de recaídas}

Objetivo. Que se incorporen conductas antidepresivas y las contemplen como hábito.

Puntos de la sesión

Monitoreo de la abstinencia.

Revisión de las actividades programadas.

Trabajo con el cuadernillo de prevención de recaída.

Aplicación de las escalas de evaluación y medición de CO exhalado.

Materiales necesarios para la sesión

Formato de programación de actividades (ver Martell et al., 2010).

Cuadernillo del plan post-terapia (adaptado de Martell et al., 2010).

Técnicas, ejercicios y procedimientos

Monitoreo de la abstinencia. Al iniciar la sesión se valoran los esfuerzos para dejar de fumar y lo que han logrado hasta ahora, los beneficios percibidos y las desventajas de dejar de fumar, las razones por las que decidieron dejar de fumar, y los efectos secundarios relacionados con el uso del fármaco para dejar de fumar. Asímis- 
mo, se discuten las situaciones de alto riesgo durante la semana y las formas de enfrentarlas.

Revisión de las actividades programadas. Se revisa la realización de las actividades planteadas en la sesión anterior, y las barreras en caso de no completarlas. Se apoya del anexo 2 , en donde se preguntan aspectos de olvido, dificultad, comprensión y conflicto de actividades. Se debe revisar si realmente las actividades que escogieron son viables o si se deben modificar. Un aspecto distintivo de las sesiones anteriores es que se plantean la duración a largo plazo de esas actividades y el resumen de todas las actividades incorporadas hasta ahora en el repertorio conductual.

Trabajo con el cuadernillo de prevención de recaída. Con apoyo del material (ver Martell et al. 2010), se resumen las estrategias que hasta ahora han sido efectivas para ayudar a permanecer en acción. Se evalúan además los contextos que aumentan la vulnerabilidad a la ansiedad, depresión y consumo de tabaco y se describen las conductas que contribuyen a que continúe el ciclo. Por último, se evalúan qué conductas antidepresivas necesitan mantenerse o incrementarse. Se incentiva a escribir en el cuadernillo para que sea llevado como recordatorio a casa y saber qué hacer para seguir incorporando actividades o conductas antidepresivas.

Aspectos que debe cubrir el terapeuta

Enfatizar los logros de cada participante.

Enfatizar los recursos y herramientas desarrolladas hasta ahora y cómo contribuye a mantenerse sin consumo.

Estilo y actitud terapéutica que deben cubrirse
Mantener la estructura de la sesión como guía.

Promover a los participantes a la acción.

Validar la experiencia del grupo.

Trabajar en colaboración con los miembros del grupo.

Reforzar los esfuerzos de los miembros del grupo.

Utilizar el refuerzo en vivo como una fuente para promover a la acción.

Escuchar con atención el relato del grupo.

Utilizar las palabras de las participantes para hablar sobre los síntomas de depresión y ansiedad.

Expresar aceptación, empatía y autenticidad.

Realizar preguntas abiertas y congruentes con la intervención.

Utilizar el humor con delicadeza y habilidad, en pro de la situación terapéutica del grupo (de ser necesario).

Trabajar en colaboración con el grupo.

Resolver las dudas del grupo.

Mostrar una comunicación directa, clara y precisa.

Tener una actitud motivadora ante el grupo.

\section{Discusión}

En este artículo se ha presentado con detalle el programa basado en la AC para el tratamiento del consumo tabáquico que tienen asociado síntomas ansiosos o depresivos. Se ha defendido que la $\mathrm{AC}$ es un tratamiento psicológico menos complicado que el paquete completo de la Terapia Cognitivo Conductual (TCC), y puede ser administrado por trabajadores de salud mental con un entrenamiento menos intensivo y costoso, sin menor efecto que la TCC, tanto en modalidad individual como 
Tabla 1.

Resumen de las sesiones grupales de AC para el abandono del tabaco

\begin{tabular}{|c|c|c|}
\hline Sesión & Punto central a trabajar en sesión & Aspecto clave para trabajar \\
\hline 1 & $\begin{array}{l}\text { Se presenta el grupo y se establecen los acuerdos } \\
\text { terapeuta-paciente. Se explica el fundamento } \\
\text { clínico de la intervención y se presenta el modelo } \\
\text { a través del análisis funcional. }\end{array}$ & $\begin{array}{l}\text { Análisis función al de la conducta, fundamentado } \\
\text { en el Modelo de Activación Conductual, de } \\
\text { Martell et al. (2010) }\end{array}$ \\
\hline 2 & $\begin{array}{l}\text { Analizar el registro de consumo, actividades } \\
\text { relacionadas y emoción que la acompaña, con } \\
\text { el fin de programar las primeras actividades. Se } \\
\text { cierra con la pauta del inicio de la abstinencia } \\
\text { y las instrucciones del fármaco para dejar de } \\
\text { fumar. }\end{array}$ & $\begin{array}{l}\text { Programación de actividades. } \\
\text { Preparación de la abstinencia. }\end{array}$ \\
\hline 3 & $\begin{array}{l}\text { Analizar las dificultades para la abstinencia que } \\
\text { inicia, revisar la realización de las actividades } \\
\text { programadas, así como las posibles situaciones } \\
\text { de riesgo y las estrategias para hacer frente. }\end{array}$ & $\begin{array}{l}\text { Seguimiento de actividades } \\
\text { Programación de nuevas y/o modificación de las } \\
\text { anteriores. }\end{array}$ \\
\hline 4 & $\begin{array}{l}\text { Monitorear la abstinencia, revisar actividades } \\
\text { programadas y planificar nuevas y/o continuar } \\
\text { con las anteriores. }\end{array}$ & $\begin{array}{l}\text { Seguimiento de actividades } \\
\text { Programación de nuevas y/o modificación de las } \\
\text { anteriores. }\end{array}$ \\
\hline 5 & $\begin{array}{l}\text { Monitorear la abstinencia, revisar actividades } \\
\text { programadas y planificar nuevas y/o continuar } \\
\text { con las anteriores. }\end{array}$ & $\begin{array}{l}\text { Seguimiento de actividades } \\
\text { Programación de nuevas y/o modificación de las } \\
\text { anteriores. }\end{array}$ \\
\hline 6 & $\begin{array}{l}\text { Monitorear la abstinencia, revisar actividades } \\
\text { programadas y planificar nuevas y/o continuar } \\
\text { con las anteriores. }\end{array}$ & $\begin{array}{l}\text { Seguimiento de actividades } \\
\text { Programación de nuevas y/o modificación de las } \\
\text { anteriores. }\end{array}$ \\
\hline 7 & $\begin{array}{l}\text { Monitorear la abstinencia, revisar actividades } \\
\text { programadas y planificar nuevas y/o continuar } \\
\text { con las anteriores. }\end{array}$ & $\begin{array}{l}\text { Seguimiento de actividades } \\
\text { Programación de nuevas y/o modificación de las } \\
\text { anteriores. }\end{array}$ \\
\hline 8 & $\begin{array}{l}\text { Monitorear la abstinencia, revisar actividades } \\
\text { programadas y planificar nuevas y/o continuar } \\
\text { con las anteriores. }\end{array}$ & $\begin{array}{l}\text { Seguimiento de actividades } \\
\text { Programación de nuevas y/o modificación de las } \\
\text { anteriores. }\end{array}$ \\
\hline 9 & $\begin{array}{l}\text { Monitorear la abstinencia, revisar actividades } \\
\text { programadas y planificar nuevas y/o continuar } \\
\text { con las anteriores. }\end{array}$ & $\begin{array}{l}\text { Seguimiento de actividades } \\
\text { Programación de nuevas y/o modificación de las } \\
\text { anteriores. }\end{array}$ \\
\hline 10 & $\begin{array}{l}\text { Prevenir la recaída continuando con las } \\
\text { actividades antidepresivas. Prevenir algunas } \\
\text { situaciones futuras que puedan disparar el } \\
\text { consumo. }\end{array}$ & $\begin{array}{l}\text { Situaciones de riesgos futuras. } \\
\text { Planes de acción. }\end{array}$ \\
\hline
\end{tabular}


grupal (Richards et al., 2016), lo que lo convierte en una intervención especialmente útil para un problema como es la adicción a la nicotina.

Este programa, en el formato que se ha presentado se aplicó en 49 pacientes que asistieron a un tratamiento para dejar de fumar, lo cual permitió constatar la utilidad y eficacia de la $\mathrm{AC}$ en esta población con este objetivo. Los hallazgos indicaron que la AC presentó mayor efecto sobre la abstinencia sostenida al mes, y a tres meses y a seis meses se igualan al tratamiento estándar. Estos datos están en proceso de publicación. Además de su utilidad y efecto, se observaron otras ventajas.

La primera es que, a diferencia de la intervención estándar para dejar de fumar, este tratamiento no solo dirige su atención sobre el consumo de tabaco, sino además sobre la sintomatología comorbida, por lo que puede servir tanto como tratamiento único para personas con depresión y ansiedad que desean dejar de fumar, como parte de algún tratamiento estandarizado más amplio. Sin embargo, es necesario aclarar que no se trata de un conjunto de técnicas o actividades previamente establecidas, pues la AC enfatiza el proceso de cada persona y toma en cuenta el contexto, por lo que su aplicación y adaptación es especialmente flexible.

El contexto emocional es un elemento importante, puesto que dentro del grupo se comentaba que dejar de fumar se les hacía más difícil especialmente ante las situaciones de depresión y ansiedad. Ante una coyuntura que les generaba esta sintomatología, la primera estrategia de evitación a la que recurrían era al tabaco, sin darse cuenta que caían en un círculo vicioso.
Específicamente, en cuanto a las modalidades de grupo y de corto plazo, el abordaje de la AC presenta varias ventajas, incluyendo la rentabilidad y la capacidad de cubrir a más pacientes de lo que es posible con terapias individuales; además, es limitado en el tiempo y menos complicada que muchas otras intervenciones psicológicas.

Su fácil aplicación hace que la persona desarrolle un comportamiento menos ansioso por medio de la programación guiada. Desde la primera sesión se observó una preocupación constante acerca de los resultados que podrían obtener, ya que referían que habían asistido a tratamientos informativos que no les aportaban mayor aprendizaje. Se les aclaró en la primera sesión que era un proceso de acompañamiento guiado y que el terapeuta en colaboración con la paciente, iban construyendo el camino hacia la activación, lo cual les iba a servir como estrategia una vez concluyera la intervención. Esto contribuyó a disminuir su preocupación.

En la primera sesión se mencionó que la AC no solo acompaña y guía, sino que incorpora elementos compatibles con otras estrategias como la resolución de problemas para esos momentos más difíciles fuera de sesión.

De acuerdo con el modelo transdiagnóstico, también implica el aumento del control y la función del comportamiento manifiesto, un atributo que puede ser útil para restaurar la pérdida de control a menudo experimentada por pacientes con deseos de dejar de fumar.

En este sentido, al ponerla en marcha en estos pacientes con depresión y ansiedad que fuman, se observó que es necesario focalizar 
prioritariamente el consumo de tabaco como una conducta de evitación, y por lo tanto contrarrestarla con otras conductas incompatibles, siendo esto el objetivo terapéutico principal. Además, los pensamientos repetitivos o rumiaciones que perjudican el avance de la persona, se trabajan como una forma de evitación y no se modifican directamente. Esta situación se repitió constantemente en este tipo de paciente, mencionando que no podían dejar de pensar mientras intentaban realizar alguna actividad. La forma en la que se trabajó fue enfocar esas rumiaciones como una forma de evitar, se invitó a prestar atención a su respiración, al lugar en donde estaba, al momento presente, etc.

Esto llevó a atender con precaución la programación de actividades, tratando que la actividad planeada no supere la capacidad de la persona o sea muy compleja, ya que esto aumentaba el sentido de incompetencia y podría ser contraproducente. A la hora de seleccionar las actividades resultó fundamental, que la persona reconociera que éstas estarán encaminadas a recuperar el reforzamiento sin necesidad de llevar a cabo la conducta de evitación. Un aspecto importante sobre la programación, fue trabajar ampliamente las múltiples áreas de vida o valores de la persona, y así crear una vida más satisfactoria.

Este aspecto les motivaba, ya que había actividades que dejaron de hacer y algunas otras que querían incorporar, pero por alguna razón no lo habían hecho.

Ahora bien, a pesar de que la AC es una opción de tratamiento, como todas las intervenciones, no puede servir en todos los casos. Antes de aplicarlo, es necesario tener en cuenta las características de quienes participan como pacientes. Por ejemplo, en su mayoría presentaban disposición al cambio y apertura para realizar las tareas, uso del discurso en sesión como una forma de evitación, bajos recursos monetarios, percepción de falta de tiempo, comprensión, y algunas con analfabetismo.

Hay que tomar en cuenta la apertura de la persona para la realización de la tarea, ya que es más fácil que una persona con disposición a realizarla le vea mayor sentido, porque a veces no les resulta agradable el proceso activo de la intervención. En este sentido, el terapeuta debe llevar a los compromisos acordados y retomar el sentido de "hacer" como una herramienta terapéutica, incorporando la lógica discutida en la primera sesión.

Otro conflicto que se presentó con frecuencia, fue la realización de la tarea. En ese caso, fue necesario recordar que la tarea es parte no solo del trabajo en la sesión, sino parte de la activación fuera de ella, y de no realizarla, se pierde la oportunidad de aprovechar al máximo el tratamiento. Sin embargo, a veces se debía a barreras considerables para hacerlas, por eso se desarrollaba un plan en colaboración con la persona, con el fin de llevarla a cabo. Para efectos de la sesión, se motivaba a la persona a anotar algunos aspectos en sesión, pero no se completaba, porque el objetivo es que lo complete en casa.

Un problema que puede encontrarse, es que la persona no tenga los recursos necesarios para llevar a cabo las actividades que se plantean, en este caso, puede tener que moldearse en algunos casos, o requerir el aprendizaje previo de otras conductas. 


\section{Agradecimientos}

Agradecemos a los pacientes por su contribución para que pudiera llevarse a cabo la intervención. Este proyecto se llevó a cabo con el apoyo financiero de la beca otorgada por el Consejo Nacional de Ciencia y Tecnología (CoNaCyT) (México) para estudios de doctorado de la primera autora del estudio (CVU) 619204 y Número de Registro Becario 335986, así como del financiamiento económico y del equipo de investigadores del Instituto Nacional de Enfermedades Respiratorias (INER).

\section{Referencias}

Aldi, G., Bertoli, G., Ferraro, F., Pezzuto, A., y Cosci, F. (2018). Efectividad de las intervenciones farmacológicas o psicológicas para dejar de fumar en fumadores con depresión mayor o síntomas depresivos: una revisión sistemática de la literatura. Abuso de Sustancias, 39, 289-306. https://doi.org/10 $.1080 / 08897077.2018 .1439802 B u s c h$ et al. 2017

Barraca, J., \& Pérez-Álvarez, M. (2015). Activación Conductual para el tratamiento de la depresión. Madrid: Síntesis

Becoña E, \& Vázquez F. The Fasgeström test for nicotine dependence in a Spanish simple. Psychological Reports, 83, 1455-1458.

Busch, A., Tooley, E., Dunsiger, S., Chattinllion, E., Fani, J., Pagoto, S.,.. \& Borrelli, B, Khaler, Ch. (2017). Behavioral activation for smoking cessation and mood management following a cardiac event: Results of a pilot randomized controlled trial. BMC Public Health, 17. Doi: 10.1186/s12889-017-4250-7

Collado, A., Calderón, M., MacPherson, L., \& Lejuez, C. (2916). The efficacy of Behavioral Activation treatment among depressed Spanish-speaking Latinos. Journal of Consulting and Clinical Psychology, 84, 651657. Doi: $10.1037 /$ ccp0000103

Collado, A., Castillo, S., Maero, F., Lejuez, C., \& MacPherson, L. (2014). Pilot of the brief Behavioral Activation treatment for depression in Latinos with limited English proficiency: Preliminary evaluation of efficacy and acceptability. Behavior Therapy, 45, 102-115. Doi:10.1016/j.beth.2013.10.001

Cooper, J., Borland, R., Mckee, S., Yong, H., \& Dugue, P. (2016). Depression motivates quit attempts but predicts relapse: Differential findings for gender from the International Tobacco Control Study. Addiction, 111, 1438-1447. Doi: 10.1111/add.13290

Dimidjian, S., Hollon, S. D., Dobson, K. S., Kohlenberg, R. J., Addis, M. E., Gallop, R. J.,... Jacobson, N. (2006). Randomized trail of behavioral activation, cognitive therapy, and antidepressant medication in the acute treatment of adults with major depression. Journal of Consulting \& Clinical Psychology, 74, 658-670. https://doi.org/10.1037/0022006X.74.4.658

Dobson, K. S., Hollon, S. D., Dimidjian, S., Schmaling, K. B., Kohlenberg, R. J., Gallop, R. J.,... \& Jacobson, N. S. (2008). Randomized trial of behavioral activation, cognitive therapy, and antidepressant medication in the prevention of relapse and recurrence in major depression. Journal of Consulting and Clinical Psychology, 76, 468-477. https://doi. org/10.1037/0022-006X.76.3.468

Ferster, C. B. (1973). A functional analysis of depression. American Psychologist, 28, 850870.

González,D., Reséndiz, A., Reyes-Lagunes, I. (2015). Adaptation of the BDI-II in Mexico. Salud Mental, 38, 237-244. Doi: 10.17711/ SM.0185-3325.2015.033

Gortner, E. T., Gollan, J. K., Dobson, K. S., \& Jacobson, N. S. (1998). Cognitive-behavioral treatment for depression: Relapse prevention. Journal of Consulting and Clinical Psychology, 66, 377-384.

Jacobson, N., Dobson, K. Truax, P., Addis, M., Koerner, K., Gollan, J., ... Prince, S. E. (1996). A component analysis of cognitivebehavioral treatment for depression. Journal of Consulting and Clinical Psychology, 64, 295-304.

Kanter, J., Santiago-Rivera, A., Santos, M., Nagy, G., López, M., Diéguez Hurtado, G., \& West, P. (2014). A randomized hybrid 
efficacy and effectiveness trial of Behavioral Activation for Latinos with depression. Behavior Therapy, 46, 177-92. http://dx.doi. org/10.1016/j.beth.2014.09.011

Lejuez, C., Hopko, D., Acierno, R., Daugthers, S., \& Pagoto, S. (2011). Ten year revision of the brief Behavioral Activation treatment for depression: Revised treatment manual. Behavior Modification, 35, 111-161. Doi: 10.1177/0145445510390929

Leventhal, A., \& Zvolensky, M. (2015). Anxiety, depression, and cigarette smoking: A transdiagnostic vulnerability framework to understanding emotion-smoking comorbidity. Psychological Bulletin, 141, 176212. http://dx.doi.org/10.1037/bul0000003

Lewinsohn, P. M. (1974). A behavioral approach to depression. En R. M. Friedman, \& M. M. Katz (Eds.), The psychology of depression: Contemporary theory and research (pp. 157185). Nueva York: John Wiley.

MacPherson, L., Tull, M., Matusiewicz, A., Rodman, S., Strong, D., Kahler, C., \& Lejuez, C. W. (2010). Randomized controlled trial of behavioral activation smoking cessation treatment for smokers with elevated depressive symptoms. Journal of Consulting and Clinical Psychology, 78, 5561. MacPherson, L., Tull, M., Matusiewicz, A., Rodman, S., Strong, D., Kahler, C., \& Lejuez, C. W. (2010). Randomized controlled trial of behavioral activation smoking cessation treatment for smokers with elevated depressive symptoms. Journal of Consulting and Clinical Psychology, 78, 55-61.

Mckay, J. (2016). Making the hard work of recovery more attractive for those with substance use disorders. Addiction, 112, 751-757. Doi: 10.1111/add.13502

Martell, C. R., Dimidjian, S., \& Herman-Dunn, R. (2010). Behavioral Activation for Depression: A Clinician's Guide. New York: Guilford.

Martínez-Vispo, C., Martínez, Ú., López-Durán, A., Fernández del Río, E. \& Becoña, E. (2018). Effects of behavioural activation on substance use and depression: A systematic review. Substance Abuse Treatment, Prevention, and Policy, 13:36. https://doi. org/10.1186/s13011-018-0173-2
Mathew, A., Hogarth, L., Leventhal, A., Cook, J., \& Hitsman, B. (2017). Cigarette smoking and depression comorbidity: Systematic review and proposed theoretical model. Addiction. 112, 401-412. Doi:10.1111/add.13604

Organización Mundial de la Salud (2019). InformedelaOMS sobrelaepidemiamundial del tabaquismo, 2019. Ginebra (Suiza): Autor. Recuperado de https://apps.who.int/ iris/bitstream/handle/10665/326072/WHONMH-PND-2019.5-spa.pdf .

Richards, D., Ekers, D., McMillan, D., Taylor, R., Byford, S., Warren, F., ... \& Gilbody, S. (2016). Cost and outcome of Behavioural Activation versus Cognitive Behavioural Therapy for depression (COBRA): A randomised, controlled, non-inferiority trial. Trials, 21, 871-880. Doi: 10.1186/1745-6215-15-29

Robles, R., Varela, R., Jurado, S., \& Páez, F. (2001). Versión mexicana del Inventario deAnsiedad de Beck: propiedades psicométricas. Revista Mexicana de Psicología, 18, 211-218.

Sánchez, J., Barraca, J., Mora, E., \& Reyes, M. (2018). Propiedades psicométricas de la Escala de Activación Conductual para la Depresión (BADS) en una muestra mexicana. Clínica y Salud, 29, 151-155.

Secades-Villa, R., Vallejo-Seco, G., GarcíaRodríguez, O., López-Núñez, C., Weidberg, S., \& González-Roz, A. (2015). Contingency management for cigarette smokers with depressive symptoms. Experimental and Clinical Psychopharmacology, 23, 351-360. https://doi.org/10.1037/pha0000044

Shared, H. (2016). Effectiveness of behavioral activation group therapy on attributional styles, depression, and quality of life in women with breast cancer. Journal of Fundamentals of Mental Health, 18, 179-88.

Villatoro-Velázquez, J. A., Resendiz-Escobar, E., Mujica-Salazar, A., Bretón-Cirett, M., Cañas Martínez, V., Soto-Hernández, I.,... MendozaAlvarado, L. (2017). Encuesta Nacional de Consumo de Drogas, Alcohol y Tabaco 20162017: Reporte de tabaco. Instituto Nacional de Psiquiatría Ramón de la Fuente Muñiz, Instituto Nacional de Salud Pública, Comisión Nacional Contra las Adicciones, Secretaría de Salud. México: INPRFM. 


\section{Anexo 1}

Monitoreo de consumo de tabaco y actividades. Registro de Placer (P), Valor (V) y Competencia (C)

Nombre: Desde el: al

Instrucciones: En el siguiente cuadro anota la cantidad de cigarros y la actividad que estabas realizando en ese momento. Además de registrar la actividad, anota el grado de Placer (P), el grado de Valor (V) y la sensación de Competencia (C), que experimentaste cuando estabas realizando cada actividad. El Placer, el Valor y la Competencia son valorados entre 1 y 10 , donde "1" equivale a un nivel "bajo" y"10" significa un nivel "alto".

\begin{tabular}{|c|c|c|c|c|c|c|c|}
\hline Horario & Lunes & Martes & Miércoles & Jueves & Viernes & Sábado & Domingo \\
\hline $5: 00-7: 00$ & $P=\underset{C=}{V=}$ & $P=\underset{C=}{V=}$ & $\mathrm{P}=\underset{C=}{\mathrm{V}=}$ & $\mathrm{P}=\underset{C=}{\mathrm{V}=}$ & $P=\underset{C=}{V=}$ & $P=\underset{C=}{V=}$ & $\mathrm{P}=\underset{\mathrm{C}=}{\mathrm{V}=}$ \\
\hline 7:00-9:00 & $\mathrm{P}=\underset{C=}{\mathrm{V}=}$ & $\mathrm{P}=\underset{\mathrm{C}=}{\mathrm{V}=}$ & $\mathrm{P}=\underset{\mathrm{C}=}{\mathrm{V}=}$ & $\mathrm{P}=\underset{\mathrm{C}=}{\mathrm{V}=}$ & $\mathrm{P}=\underset{\mathrm{C}=}{\mathrm{V}=}$ & $\mathrm{P}=\underset{\mathrm{C}=}{\mathrm{V}=}$ & $\mathrm{P}=\underset{\mathrm{C}=}{\mathrm{V}=}$ \\
\hline 9:00-11:00 & $\mathrm{P}=\underset{\mathrm{C}=}{\mathrm{V}=}$ & $\mathrm{P}=\underset{\mathrm{C}=}{\mathrm{V}=}$ & $\mathrm{P}=\underset{\mathrm{C}=}{\mathrm{V}=}$ & $\mathrm{P}=\underset{\mathrm{C}=}{\mathrm{V}=}$ & $\mathrm{P}=\underset{\mathrm{C}=}{\mathrm{V}=}$ & $\mathrm{P}=\underset{\mathrm{C}=}{\mathrm{V}=}$ & $\mathrm{P}=\underset{\mathrm{C}=}{\mathrm{V}=}$ \\
\hline 11:00-13:00 & $P=\underset{C=}{V=}$ & $P=\underset{C=}{V=}$ & $P=\underset{C=}{V=}$ & $\mathrm{P}=\underset{\mathrm{C}=}{\mathrm{V}=}$ & $P=\underset{C=}{V=}$ & $\mathrm{P}=\mathrm{C}=^{\mathrm{V}=}$ & $\mathrm{P}=\underset{\mathrm{C}=}{\mathrm{V}=}$ \\
\hline 13:00-15:00 & $P=\underset{C=}{V=}$ & $P=C_{C=}^{V=}$ & $P=C_{C=}^{V=}$ & $P=\underset{C=}{V=}$ & $P=\underset{C=}{V=}$ & $P=\underset{C=}{V=}$ & $\mathrm{P}=\underset{\mathrm{C}=}{\mathrm{V}=}$ \\
\hline $15: 00-17: 00$ & $P=\underset{C=}{V=}$ & $P=\underset{C=}{V=}$ & $P=\underset{C=}{V=}$ & $P=\underset{C=}{V=}$ & $P=\underset{C=}{V=}$ & $P=\underset{C=}{V=}$ & $\mathrm{P}=\underset{\mathrm{C}=}{\mathrm{V}=}$ \\
\hline 17:00-19:00 & $\mathrm{P}=\underset{\mathrm{C}=}{\mathrm{V}=}$ & $\mathrm{P}=\underset{\mathrm{C}=}{\mathrm{V}=}$ & $\mathrm{P}=\underset{\mathrm{C}=}{\mathrm{V}=}$ & $\mathrm{P}=\underset{\mathrm{C}=}{\mathrm{V}=}$ & $\mathrm{P}=\underset{\mathrm{C}=}{\mathrm{V}=}$ & $\mathrm{P}=\underset{\mathrm{C}=}{\mathrm{V}=}$ & $\mathrm{P}=\underset{\mathrm{C}=}{\mathrm{V}=}$ \\
\hline 19:00-21:00 & $\mathrm{P}=\underset{C=}{\mathrm{V}=}$ & $\mathrm{P}=\underset{C=}{\mathrm{V}=}$ & $\mathrm{P}=\underset{C=}{\mathrm{V}=}$ & 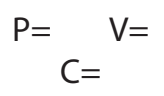 & $\mathrm{P}=\underset{\mathrm{C}=}{\mathrm{V}=}$ & $\mathrm{P}=\underset{\mathrm{C}=}{\mathrm{V}=}$ & $P=\underset{C=}{V=}$ \\
\hline
\end{tabular}

Material desarrollado por Sánchez, J.; \& Reyes, M.. (2017). 


\section{Anexo 2}

\section{Preguntas que considerar al revisar la tarea.}

¿Fue un problema de olvido?

¿Fue un problema de comprensión?

Tipo de problema

¿Decidieron hacer la tarea o lo olvidaron? ron hacerlo o algo ocurrió?

Si usaron recordatorio y aun así no funcionó, ¿Por qué creen que no funcionó?

¿Qué hacer?

Trabajar con recordatorios (control de estímulos)

¿Qué se puede hacer distinto si vuelve a ocurrir

Modificar el recordatorio

Recordar que se trabaja en función a un plan y no a un estado de ánimo y que la única forma de que funcione

Si lo recordaron, pero aun así decidieron no hacerlo la terapia es haciendo.

Se trabaja con el análisis del modelo previo y la pasividad/evitación mantiene la depresión

¿Fue un problema de conflicto de actividades?

\section{Tipo de problema}

¿Has hecho este tipo de cosas antes o esto es nuevo para ti?

¿Tenías claro lo que tenías que hacer antes de empezar, iniciaste y luego te estancaste?

\section{¿Qué hacer?}

Solución de problemas

Aclarar dudas

Se regresa a la tarea para romper el ciclo de pasividad, no para sentirse mejor.

¿Fue un problema de implementación (habilidad) ¿Es necesario desglosar la actividad en

\section{Tipo de problema}

¿Será que para realizar esta actividad necesitan mucho tiempo, y dejan de hacer otras obligaciones?

¿Qué actividad se dejaría de realizar, si decidieran hacer la actividad que seleccionaron?

¿Es posible prescindir de estas obligaciones, para realizar la actividad que escogieron y que pueden darles mayor sensación de satisfacción en un futuro cercano?

segmentos más pequeños?

Material desarrollado por Sánchez, J.; \& Reyes, M.. (2017).

Tipo de problema

¿Es muy compleja la actividad?
¿Qué hacer?

Atender el tiempo necesario mínimo para realizar la actividad, considerándolo en la próxima programación de las actividades

Estimular a la elección de la actividad que pueda aumentar el valor, placer y competencia a corto plazo.

¿Cómo se sentirían si dejaran de hacer la otra obligación y decidieran hacer la actividad seleccionada en la programación?

Análisis de ventajas y desventajas
¿Qué hacer?

Desglosar la actividad en pequeños pasos y comenzar por el primero. 


\section{Anexo 3}

\section{Contrato terapéutico}

En la Ciudad de México, el día de de , me encuentro en calidad de paciente que desea dejar de fumar. Se reúnen las siguientes personas: Psicoterapeuta cuyo nombre es y por otra parte paciente, cuyo nombre es , para establecer los compromisos durante el tratamiento:

\section{CLÁUSULAS PARA EL/LA TERAPEUTA}

Se COMPROMETE a proporcionar apoyo psicológico de acuerdo con los principios de la ética profesional previstos en el Código Ético del Psicólogo en México.

Se COMPROMETE a orientar con claridad fijando objetivos terapéuticos claros, orientándolo y asesorándolo durante el tiempo que dure el tratamiento.

Se COMPROMETE a que la información será confidencial.

Se COMPROMETE a asistir puntualmente a las citas que se haya señalado con anterioridad y si por algún problema de fuerza mayor no pudiese asistir a la cita, avisará al paciente con 24 horas de anticipación como mínimo.

\section{CLÁUSULAS PARA EL/LA PACIENTE}

Se COMPROMETE a realizar todos los esfuerzos necesarios para lograr un cambio, con el fin de lograr su bienestar físico y mental.

Se COMPROMETE a realizar esfuerzos para realizar las tareas asignadas para casa.

Se COMPROMETE a esforzarse por asistir a todas las sesiones del programa.

Se COMPROMETE a asistir con puntualidad. En caso de no poder acudir, avisará con anticipación. 